\author{
WALDEN \\ UNIVERSITY \\ A higher degree. A higher purpose.
}

Walden University ScholarWorks

Walden Dissertations and Doctoral Studies

2018

\title{
A Clinical Practice Guideline for Pain Management in the Post Anesthesia Care Unit
}

Susan Mogan

Walden University

Follow this and additional works at: https://scholarworks.waldenu.edu/dissertations

Part of the Nursing Commons

This Dissertation is brought to you for free and open access by the Walden Dissertations and Doctoral Studies Collection at ScholarWorks. It has been accepted for inclusion in Walden Dissertations and Doctoral Studies by an authorized administrator of ScholarWorks. For more information, please contact ScholarWorks@waldenu.edu. 


\title{
Walden University
}

\author{
College of Health Sciences
}

This is to certify that the doctoral study by

Susan Mogan

has been found to be complete and satisfactory in all respects, and that any and all revisions required by the review committee have been made.

\author{
Review Committee \\ Dr. Mary Catherine Garner, Committee Chairperson, Nursing Faculty \\ Dr. Geri Schmotzer, Committee Member, Nursing Faculty \\ Dr. Corinne Wheeler, University Reviewer, Nursing Faculty
}

Chief Academic Officer

Eric Riedel, Ph.D.

Walden University

2018 


\begin{abstract}
A Clinical Practice Guideline for Pain Management in the Post Anesthesia Care Unit by

Susan Mogan
\end{abstract}

MSN, Western Connecticut University, 2012

BSN, Pace University, 1995

Project Submitted in Partial Fulfillment

of the Requirements for the Degree of

Doctor of Nursing Practice

Walden University

December 2018 


\begin{abstract}
Ineffective pain management in the post anesthesia care unit (PACU) increases patients' risk of adverse effects including decreased mobility, infection, chronic pain, depression, cardiopulmonary complications, increased length of stay, insomnia, fatigue, and overall decrease in quality of life. The PACU in a community hospital did not provide an evidence-based pain management guideline for nurses treating postoperative patients, resulting in nurses' concerns about providing pain management. The purpose of this project was to translate evidence on pain management into an evidence-based guideline for improved nursing practice in a PACU. Evidence was obtained from a detailed literature search using multiple databases and professional organizations' guidelines. Nursing practice guidelines were developed and evaluated by 3 expert panelists using the Agree II guidelines. The panelists selected included; Two anesthesiologists, one who is trained in pain management and is also a pharmacist. The third expert is a practicing nurse practitioner in an acute setting who is also a surgical first assist and the associate director of robotics. The panel endorsed the guidelines for advancement through the hospital's review committees. Implementation of the evidence-based pain management guideline in the PACU might provide nurses with tools to guide their interventions and improve patient outcomes. Social changes resulting from the use of evidence-based pain management guidelines include decreased time to opiate administration, decreased adverse effects, improved assessment of pain, and an increase in the number of patients who receive proper pain management.
\end{abstract}


A Clinical Practice Guideline for Pain Management in the Post Anesthesia Care Unit by

Susan Mogan

MSN, Western Connecticut University, 2012

BSN, Pace University, 1995

Project Submitted in Partial Fulfillment

of the Requirements for the Degree of

Doctor of Nursing Practice

Walden University

December 2018 


\section{Dedication}

I dedicate this project to my beautiful mom, Sheila, and my best friend, Martha, who are now angels in heaven. Sheila and Martha encouraged me to achieve my dream and calling to become a nurse. Being a nurse has enriched my life, and I have been blessed and lucky to be allowed to care for so many in their time of need. 


\section{Acknowledgments}

I want to first and foremost thank my mom, Sheila, without whose love, support, and encouragement I never would have become a nurse or the person I am today. Next, my wonderful husband, Patrick, and my three children, Pat, Sarah, and Emi, without whose love and support I could never have achieved all that I have. My dad, who also supported me and continues to encourage me throughout my career. My loving mother and father in law who have helped me throughout all of my education. I love you all. 
Table of Contents

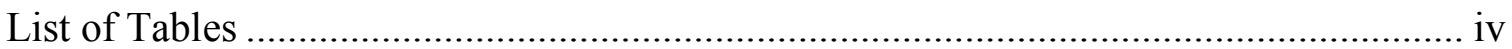

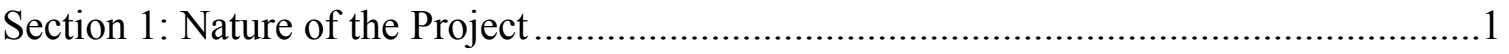

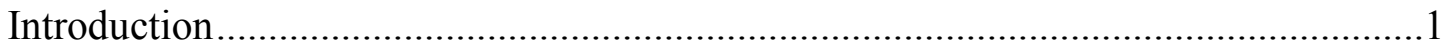

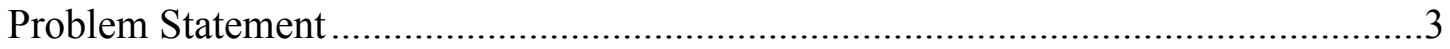

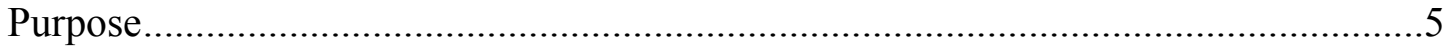

Nature of the Doctoral Project ...............................................................................

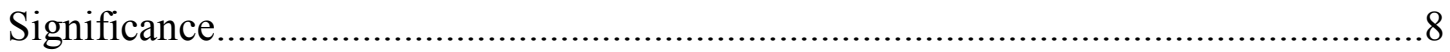

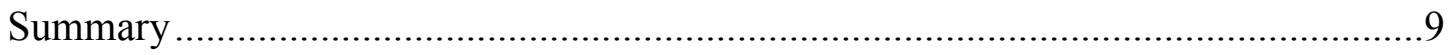

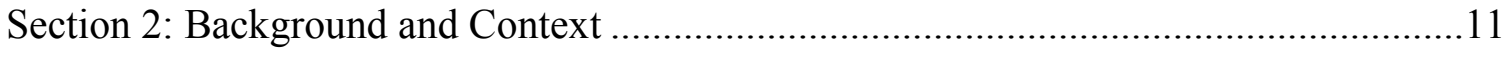

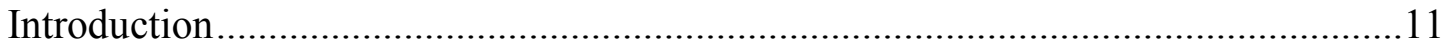

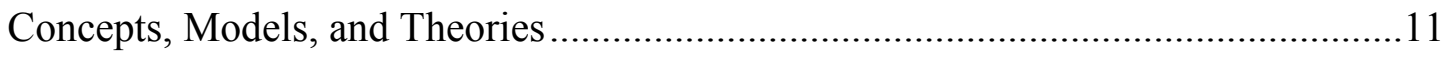

Pain Management Protocols and Assessment of Pain in the PACU.................... 12

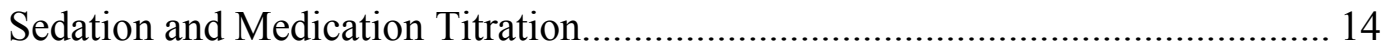

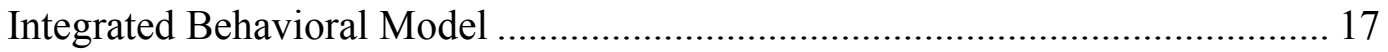

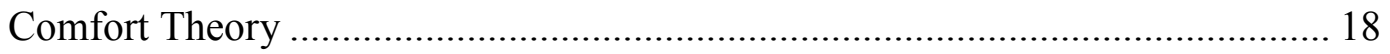

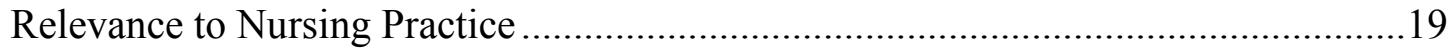

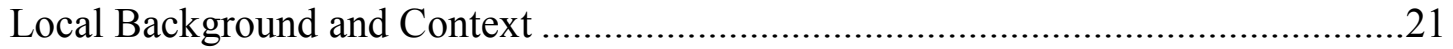

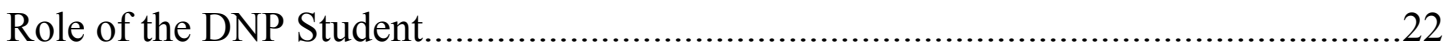

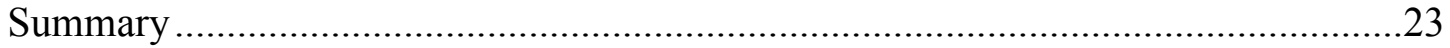

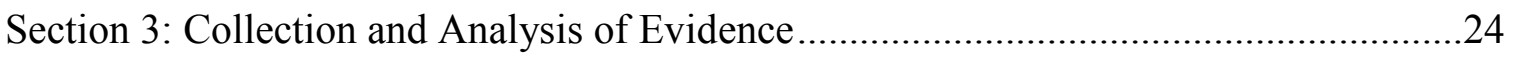

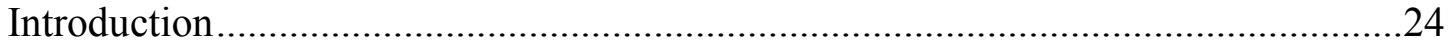




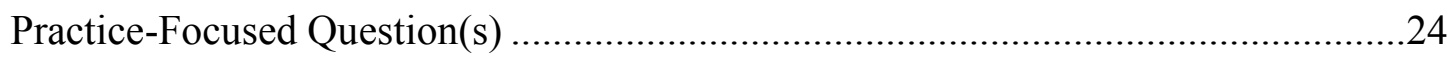

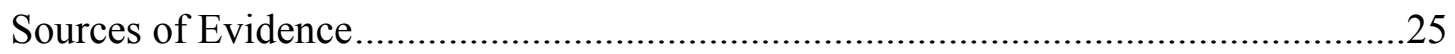

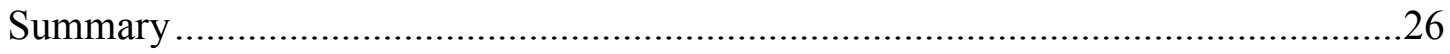

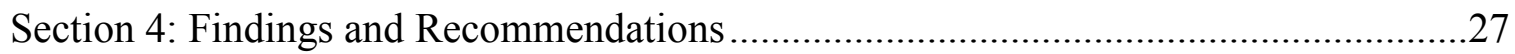

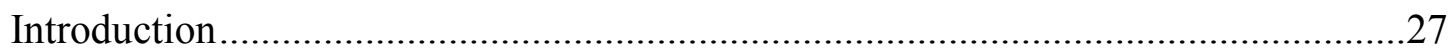

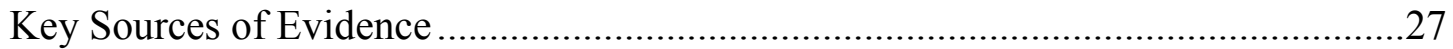

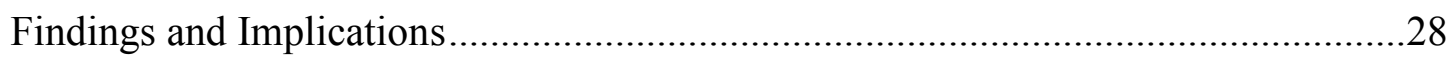

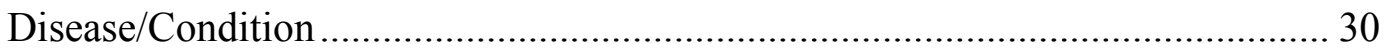

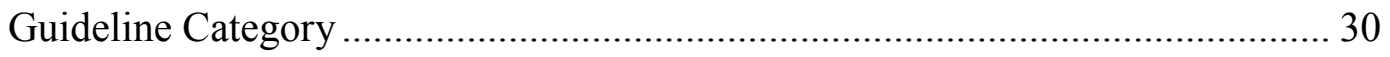

Adequate Pre- and Postoperative Assessment-Criteria ....................................... 30

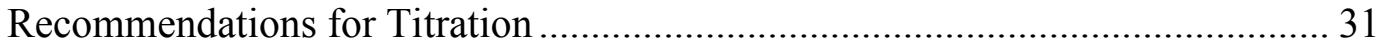

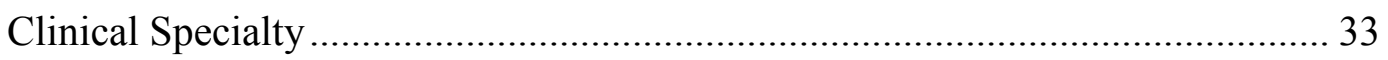

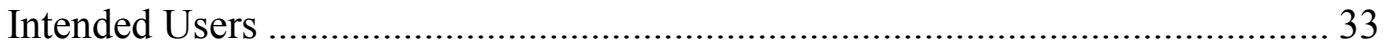

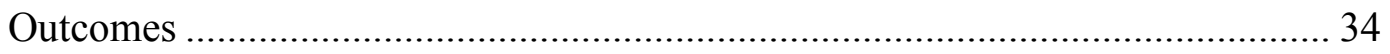

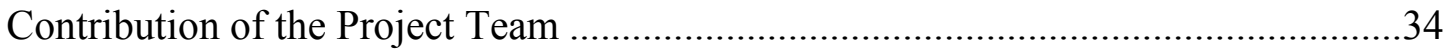

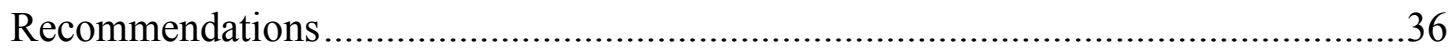

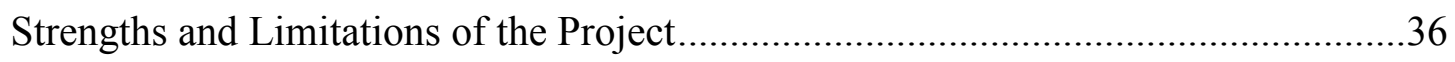

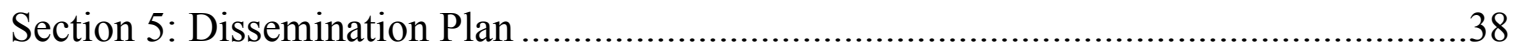

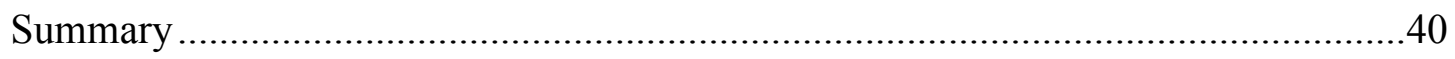

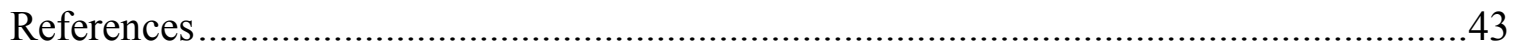

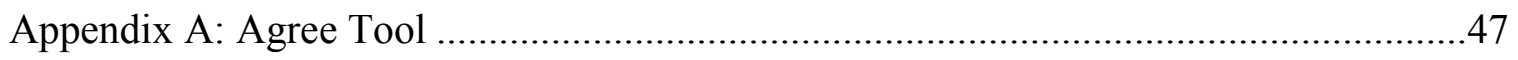

Appendix B: Evidence-Based Pain Management Algorithm .............................................48 
Appendix C: Pasero Opioid-Induced Sedation Scale (POSS) ......................................49

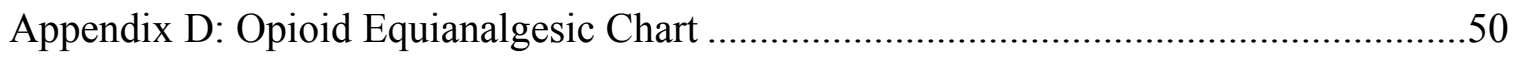

Appendix E: Evidence-Based Pain Management Guidelines for the Postanesthesia

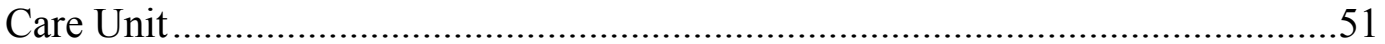




\section{List of Figures}

Figure 1. Results of the Agree tool provided by the expert panel ......................................35 
Section 1: Nature of the Project

Introduction

The identified problems in a post anesthesia care unit (PACU) in a community hospital include the concurrent use of pain management tools versus the use of the recommended numeric pain scale (NPS), stacking opiates versus titration of analgesics and opiates, and lack of an opioid sedation scale as recommended by the Joint Commission. This quality improvement project involved the development of an evidence-based pain management guideline to be used in the PACU. This guideline includes the proper use of the NPS and the Face, Legs, Activity, Cry, Consolability (FLACC) scale when the NPS is not appropriate. The guideline also includes an opioid sedation scale, specifically the Pasero Opioid Sedation Scale (POSS). The guideline guides nurses through the proper titration of analgesics and provides an algorithm that was created and will be implemented along with the guideline, pending final organizational approval.

The World Health Organization (WHO) developed an analgesic ladder in 1986 to assist healthcare professionals with the proper titration of analgesics for pain management (Ballantyne, Kalso, \& Stannard, 2016). Proper titration of analgesics, including opiates, is important for safe and effective pain management. Evidence supports the recommendation that the titration of analgesics, including opioids, be done in the PACU. The evidence-based pain management guideline developed for this project included the proper titration of opioids as described in the literature. 
The use of evidence-based pain management not only will lead to improvements in the PACU, but also will have positive social implications related to the use of opiates within the hospital and the community. There are two phenomena related to opioids known as the opioid epidemic and opiophobia (Glowacki, 2015). These two phenomena are barriers to effective pain management (Glowacki, 2015). Last year, 64,000 people died from opioid overdoses in what has been described as an opioid epidemic (National Institute on Drug Abuse, 2017). The opioid epidemic has led to a second opioid phenomenon called opiophobia (Reville \& Foxwell, 2014). Opiophobia is the fear of opiates (Reville \& Foxwell, 2014).

Both opioid phenomena are contributing factors in ineffective pain management (Glowacki, 2015). Another contributor to ineffective pain management is lack of education on evidence-based pain management among healthcare professionals (Pasero, Quinlan-Colwell, Rae, Broglio, \& Drew, 2016). There is a documented lack of education and knowledge among healthcare providers, including nurses and doctors, on evidencebased pain management (Pasero et al., 2016). The fear of harm from opiates, lack of knowledge and education on evidence-based pain management, and the "opioid epidemic" are all barriers to the proper use of evidence-based pain management. Education, protocols, guidelines and expert opinion must be integral parts of an effort to translate evidence-based pain management into practice to achieve improved patient outcomes (White, Dudley-Brown, \& Terhaar, 2016). The development and implementation of an evidence-based pain management guideline will help to provide effective evidence-based pain management and improve patient outcomes. 


\section{Problem Statement}

The PACU in a community hospital is not currently using an evidence-based pain management guideline to treat postoperative patients. The WHO and the American Pain Society (APS) recommend titrating analgesics and opioids for safe and effective treatment of pain. The current practice in this PACU does not include the titration of opioids; rather, the stacking of opiates is the current practice. Proper titration of an opioid is to increase the dose by $50-100 \%$ of the ineffective dose to be given once the peak action is reached (Ingram, Deming, \& Bock, 2015). Opioids should be titrated upward or downward according to effectiveness coupled with adverse effects (Chou, Gordan, De Leon-Casasola, Rosenberg, \& Bickler, 2015). The current assessment of pain does not include an opioid sedation scale as recommended by the literature. Lack of use of an evidence-based pain management guideline has led PACU nurses to make frequent calls to anesthesiologists and the pain management nurse practitioner (NP) for assistance with poor pain relief and patient complaints of pain after discharge from the PACU. The PACU nurses express frustration due to not being provided with the pain management guidelines that they need to provide the best care to their patients. The present care management strategies used by the PACU nurses are based on habit and past practice rather than evidence. Moreover, these strategies reflect that an evidence-based guideline has not been provided to assist PACU nurses with pain management, resulting in a gap in nursing practice.

The literature indicates that there is a lack of knowledge among nurses on evidence-based pain management and a lack of understanding of the pharmacokinetics of 
opiates (Pasero et al., 2016). Nurses in the PACU are presently using the FLACC scale for adult patients who are capable of scaling their pain using the NPS. It has been found through interviewing the PACU nurses that they feel "compelled" to assess both the NPS and the FLACC scale because both pain scales are listed as options when medicating for pain. PACU nurses have expressed difficulty differentiating between sedation and sleepiness, which inhibits them from medicating patients who complain of pain, due to fear of adverse effects (Pasero et al., 2016). This difficulty has been expressed by the nurses themselves and the pain management NP. Myths and misconceptions about pain management often affect PACU nurses' attitudes toward effective pain management (Pasero et al., 2016). Practicing evidence-based pain management in the PACU can improve pain relief in the PACU (Chou et al., 2015).

Evidence-based pain management guidelines have demonstrated decreased time to opiate administration, decreased adverse effects, improved assessment of pain and an increase in the number of patients who receive proper pain management (Ridderikhof, et al., 2017) Pain management guidelines decrease a patient's length of stay, thus reducing healthcare costs (Douma, Drake, O'Dochartaigh, \& Smith, 2016). Implementing evidence-based pain management improves patient satisfaction (Glowacki, 2015). Ineffective pain management in the PACU increases patients' risk of adverse effects, which include decreased mobility, infection, chronic pain, depression, cardiopulmonary complications, increased length of stay, insomnia, fatigue, and an overall decrease in quality of life (Glowacki, 2015). It has been noted that ineffective pain management can increase morbidity and mortality (Malcolm, 2015). Older patients 
are at increased risk of ineffective pain management secondary to ageism and nurses' attitudes toward medicating the elderly for pain issues (Malcolm, 2015). The use of evidence-based practice is important in the assessment and management of pain by the PACU nurse.

\section{Purpose}

The gap in practice is the lack of evidence-based management of pain in the PACU. The gap in practice was addressed through the development of an evidence-based pain management guideline for use in the PACU in a community hospital. In this facility, there is a pain management NP who assists nurses in the PACU with evidencebased pain management. The PACU nurses do not have guidelines to follow and often must contact the NP due to ineffective postoperative orders. The nurses contact the pain management NP when a patient's pain is not being relieved with the currently available interventions. This NP uses evidence-based pain management strategies to improve patient outcomes. The current pain management order sets for the PACU do not include the titration of opiates as per current evidence, and currently there are no clinical practice guideline to assist the nurses in the care of the postoperative patient.

The current practice is stacking opiates. This consists of giving the same dose of an opiate more than once if the first dose is ineffective. The evidence suggests that opiates should be slowly titrated upward or downward depending on their effect (Ballantyne et al., 2016). If the patient continues to complain of pain or the dose was ineffective, then titrating the dose upward is necessary (Ballantyne et al., 2016). An 
opioid sedation scale, as recommended by the Joint Commission, is not provided to the nurses for assessment (Davis, et al., 2017).

The Centers for Medicare and Medicaid Services take the stance that all patients have the right to effective pain management and that they also have the right to participate in their plans of care (Harding \& Flynn-Harding, 2014). Pain management order sets are recommended by the Institute of Safe Medication Practices (ISMP; Pasero et al., 2016). The recommendations include the use of pain management order sets to reduce the need to call providers for clarification of orders. According to the recommendations, order sets should be clear and specific, should be objective, and should omit any range orders that are not attached to objective measures (Pasero et al., 2016). Inadequate evidence-based pain management education is a limitation among health care professionals (Pasero et al., 2016).

Evidence also suggests that the use of evidence-based pain management interventions for acute pain will decrease the risk of the progression to chronic pain (Pierik, et al., 2016). In addition to a knowledge deficit, poor interdisciplinary communication, negative attitudes, and misconceptions contribute to ineffective pain management and the need for evidence-based pain management order sets and pain management guidelines (Pierik, et al., 2016). Pain management guidelines and order sets have been shown to not only decrease the time to administration of pain medicines, but also increase the number of patients who receive effective analgesia (Pierik, et al., 2016). Barriers to the practice of effective pain management among health care providers include fears of adverse effects of opioids and nurses not believing patients' own pain 
assessments (Drake \& Williams, 2017). Pain is undertreated in the PACU, and ineffective pain management increases post-surgical patients' risks of problems in the nervous system leading to chronic pain and related issues, as well as poorer quality of life with unwarranted psychological suffering (Hayes \& Gordan, 2015). The practice-focused question for this project was the following: Can an interdisciplinary team develop an evidence-based pain management guideline to improve pain management in PACU patients?

Using an evidence-based pain management guideline in the PACU will provide nurses with the tools needed to guide their interventions and improve patient outcomes. The evidence-based pain management guideline will guide nurses according to a stepwise approach, as recommended by the WHO for the proper use of analgesics (Ballantyne et al., 2016). This guideline involves titration of opioids according to the status of the patient (Ballantyne et al., 2016). The evidence-based pain management guideline involves the use of the NPS along with an opioid sedation scale as recommended by the Joint Commission (Davis, et al., 2017). With this guideline, the nurses will be guided as to whether opioids should be given, whether opioids should be titrated upward or downward, and when it is appropriate to notify a specialist.

\section{Nature of the Doctoral Project}

Sources of evidence were collected through multiple sites. Search engines used included Medline, PubMed, Science Direct, CINAHL, ProQuest Health, Cochrane, and Medical Collection. These search engines were located through the Walden University library. The years of publication that were used were 2012-present. The terms used in the 
search included pain management, pain management protocols, titrating opioids, postoperative pain, pain, opioid titration, palliative care, the comfort theory, post anesthesia care unit, and nurse practitioner.

The approach used for this project included the creation of an evidence-based pain management clinical practice guideline and an algorithm to assist the users of the guideline in visualizing the process. Once approved through all hospital quality channels, educational sessions will be provided for the nurses prior to the use of the algorithm and the implementation of the clinical practice guideline. Evaluation of the PACU nurse educational program on the use of the clinical practice guideline will be conducted using a pretest and posttest to assess the nurses' knowledge on pain management and attitudes regarding the use of the guideline.

\section{Significance}

Many stakeholders will be affected by the introduction of an evidence-based pain management guideline in the PACU. First and foremost are the post-surgical patients. Using this guideline will directly affect patients' pain management and improve outcomes. The PACU bedside nurses will also be directly affected. This guideline represents a change in their current nursing practice and may improve their knowledge on evidence-based pain management, improve their assessment skills, and provide them with comfort in practice when using opioids.

The pain management NP is a key stakeholder. The NP is the direct contact for the nurses when there is inadequate pain relief among this population. The anesthesiologists are also key stakeholders. When the pain management NP is not 
available, the anesthesiologist is called. This pain management guideline can also guide the anesthesiologist in the proper use of evidence-based pain management. The hospital as a community, and administration and senior leadership are also key stakeholders.

Evidence-based pain management and guidelines have been shown to reduce length of stay, leading to a reduction in healthcare costs and directly affecting the hospital community (Glowacki, 2015). Inadequate pain management in the acute pain patient can lead to increased risk of chronic pain, mortality and morbidity (Malcolm, 2015). Chronic pain patients frequently use emergency departments and cost the healthcare industry

more than half a billion dollars yearly (McCauley, Mercer, Barth, Brady, \& Back, 2014). Poor pain management leads to increased length of stay and admissions (Douma et al., 2016).

Improving pain management can improve patients' comfort levels, ambulation, and health outcomes. An evidence-based pain management guideline that is successfully implemented in the PACU can be transferred for use in multiple units within the hospital such as the emergency department, medical-surgical units, critical care units, and palliative and oncological units. This evidence-based pain management guideline could be adopted by the system and other hospitals across the nation.

\section{Summary}

The PACU in a community hospital does not currently use an evidence-based pain management guideline. The project developed an evidence-based pain management guideline to be used in a PACU in a community hospital. An algorithm was also developed as a visual guide to assist nurses with the use of the guideline. The gap in 
practice will be closed by providing educational sessions for the nurses on the evidencebased pain management guideline, and the guideline will be implemented after the educational sessions are completed.

Pain has been globally and historically undertreated in the PACU, and this ineffective pain management increases post-surgical patients' risks of problems in the nervous system leading to chronic pain and related issues, placing them at increased risk of a poorer quality of life with unwarranted psychological suffering (Hayes \& Gordan, 2015). Implementation of an evidence-based pain management guideline in the PACU will improve nursing care and patient outcomes. The following section addresses the concepts, models and theories used to guide this project, the project's relevance to nursing practice, the local background and context, and my role as a Doctor of Nursing Practice (DNP) student in this project. 
Section 2: Background and Context

Introduction

Ineffective pain management in the PACU increases patients' risk of adverse effects, which include decreased mobility, infection, chronic pain, depression, cardiopulmonary complications, increased length of stay, insomnia, fatigue, and an overall decrease in quality of life (Glowacki, 2015). It has been noted that ineffective pain management can increase morbidity and mortality (Malcolm, 2015). The gap in practice is the lack of evidence-based management of pain in the PACU. Specific practice problems include the use of more than one pain assessment scale at the same time, difficulty differentiating between sedation and sleeping, lack of use of an opioid sedation scale, and lack of titration of analgesics and opiates. The nurses are not provided with the tools needed for them to provide the best care for their patients. The practice focused question is the following: Can an interdisciplinary team develop an evidencebased pain management guideline to help improve pain management in PACU patients?

The gap in practice will be addressed through the development of an evidence-based pain management guideline for use in the PACU in a community hospital.

The following section addresses the concepts, models and theories used for this project; the project's relevance to nursing practice; the local background and context of the evidence; and my role as the DNP student in this project.

\section{Concepts, Models, and Theories}

The integrative behavior model was used to guide this project, along with comfort theory. The integrative behavior model may be used to evaluate a nurse's intentions 
when giving pain medications as needed (Taylor, Sheu, Chen, Glassman, \& Dake, 2017). The comfort theory describes comfort as the ease, relief, and transcendence that occur when pain is relieved (Petiprin, 2016). This project will translate evidence on pain management and the use of a guideline into the nursing practice in a PACU in a community hospital. The identified practice problems include the use of more than one pain assessment scale at the same time, difficulty differentiating between sedation and sleeping, lack of use of an opioid sedation scale, and lack of titration of analgesics and opiates. Nurses must be provided with these tools to better assist their nursing practice and improve patient outcomes.

\section{Pain Management Protocols and Assessment of Pain in the PACU}

Schroeder et al. (2016) used a single-group pre-posttest design that consisted of four phases to study how nurses' knowledge on pain management is related to patient satisfaction. The study took place in a 15-bed orthopedic unit within a 325-bed community hospital. The number of nurses included in the study was 30 . In the first phase of the study, patients received a questionnaire related to their pain experience during their recent hospitalization. During the second phase of the study, an online educational program on pain management was provided to the nurses. The third phase consisted of sending out the same pain-related questionnaire to the same number of patients as in Phase 1. In the last phase, the perceptions of the nurses were evaluated through a focus group of six nurses from the unit where this study took place. The results for the Nurse Knowledge Questionnaire improved significantly after nurses participated in the educational program. Patient satisfaction was not significantly affected. The 
results for patient satisfaction were similar prior to the nurse's educational program and after nurses' participation in the educational program.

Chou et al. (2015), as part of an expert panel in collaboration with the APS and the American Society of Anesthesiologists, developed a clinical practice guideline that consists of 32 postoperative pain management guidelines. This clinical guideline was based on a systematic review of the literature. The panel consisted of 23 members from multiple disciplines, including but not limited to anesthesia, surgery, obstetrics, pain medicine, hospital medicine, nursing, and primary care. The review of evidence took place at the Oregon Evidence-Based Practice Center and consisted of 107 systematic reviews and 858 primary studies. The studies were evaluated using the Grading Recommendations Assessment, Development, and Evaluation Working Group to grade the evidence (Chou et al., 2015). Strength (strong or weak) and quality (high, moderate, or poor) of the evidence were measured. The following guidelines are most relevant to this study:

- Recommendation 3 involves the use of a preoperative evaluation to identify any history of chronic pain, substance use disorders, and previous pain management interventions that were effective.

- Recommendation 4 involves adjustment of the pain management plan according to effects and adverse reactions.

- Recommendation 5 suggests the use of assessment and reassessment of pain management interventions, including assessment of pain and sedation. 
Barksdale, Hackman, Williams and Gratton (2016) used a retrospective chart review of patients who presented to an emergency department over a 2-year period. The study reviewed the effectiveness of the implementation of a nurse-initiated pain management protocol. The protocol involved the titration of analgesics as recommended by the WHO analgesic ladder. The study included 23,409 patients who presented to the emergency department with a complaint of pain. Of the 23,409 patients, 13,112 received pain medications. Of this 13,112 , the pain management protocol was used in 1,004 patients. The results showed that the use of an emergency department pain management protocol decreased the time between a patient's complaint of pain and the administration of pain medications. The findings were significant for the mean time between preimplementation (238 minutes) and postimplementation (168 minutes) with $p<.0001$.

Douma et al. (2016) used a computer-generated randomized, controlled evaluation of the use of nurse-initiated protocols in an emergency room, including a pain management protocol. The researchers provided 30 emergency room nurses with the protocol. The patient sample size was 143 patients for four different nurse-initiated protocols. The use of the protocol reduced the time from a patient's complaint of pain to administration of an analgesic by 186 minutes. Nurses and physicians expressed a high level of satisfaction with the use of the protocols.

\section{Sedation and Medication Titration}

Jungquist et al (2016) used a retrospective chart review to evaluate the monitoring of opioid-induced respiratory depression practices of nurses in eight hospitals. Data were collected through the electronic medical record. All patients who were provided with 
opioid analgesia via a patient-controlled-analgesia pump for at least 2.5 hours were included. Evaluation included the number of patients who were monitored as per recommended standards that were created by an expert panel. The study included comparisons of the use of sedation monitoring and the administration of naloxone, a medication used for the reversal of opioid-induced sedation. The results showed that $8.3 \%$ of patients were monitored appropriately according to the standards.

Davis et al (2017) used a retrospective study to assess the result of implementing the POSS or re-educating nurses in a facility that was already using the POSS. The researchers studied the effects of the POSS on 212 patients at six healthcare facilities. The study's purpose was to evaluate the effectiveness of the use of the POSS for increasing the identification of unintended sedation secondary to opioid administration, specifically intravenous hydromorphone. The use of naloxone, an opioid antagonist used to reverse the effects of opioids, served as an indication as to whether a patient experienced unintended sedation secondary to receiving opioids. It was found that naloxone use did not differ pre- versus postintervention. Naloxone use occurred in 1.9\% of each group with a $p=.90$. It was found that compliance with charting POSS results improved significantly postintervention, $78.1 \%$ as compared with $26.4 \%$ of the time, with a $p<.001$. It was also found that unintended sedation documentation increased after the intervention, $12.2 \%$ versus $3.8 \%$, with a $p=.04$. The findings suggest that use of the POSS provides quicker assessment and identification of unintended sedation. It also suggests that education on the use of the POSS improves nurses' knowledge of the signs 
and symptoms of unintended sedation, which leads to improved confidence in nursing care.

Jungquist, Willens, Dunwoody, Klingman, and Polomano (2014) surveyed 102 hospital representatives from 36 of the 50 United States. The data were analyzed using descriptive statistics. The researchers evaluated the use of sedation scales with the administration of opioids. The results showed that $100 \%$ of the hospitals surveyed used an opioid sedation scale. This was an increase from a previous study done 4 years prior, which showed that $64 \%$ of the same hospitals used an opioid sedation scale. The results showed that the POSS was used in most of the participating hospitals, $52.9 \%$ of the 102 . This was an increase relative to findings of the previous study done by these researchers. The researchers attributed this increase to the reliability, validity, and ease of use of the POSS.

Kobelt, Burke, and Renker (2014) used a quasi-experimental design to evaluate the implementation of the POSS in two PACU. One PACU served 7,000 patients annually, and the other served 13,300 patients each year. This study included 842 patients and 67 nurses. The nurses completed a survey that evaluated the implementation of a POSS protocol. The researchers reviewed the nurses' satisfaction with communication before and after implementation. They reviewed the nurses' perceptions of quality of care with the use of the protocol and the nurses' confidence level related to opioid administration. The results showed a significant improvement in the nurses' perception of quality of care, from 6.76 to 7.29 on a 10-point scale. Results using a paired $t$ test were $\mathrm{t}=-2.985$. There was also a significant increase in the nurses' confidence in 
opioid administration, from 7.55 to 8.59 on a 10-point scale, with a paired t-test result of $\mathrm{t}=-2.668$. The nurses' confidence in the use of the POSS to identify unintended opioid sedation and administering opioids for pain increased from 7.95 to 8.77 on a 10 -point scale, with a paired t-test result of $t=-2.673$.

\section{Integrated Behavioral Model}

Taylor et al. (2017) used a nonexperimental, cross-sectional, quantitative, anonymous survey to study how the integrated behavioral model's elements relate to the intentions of a nurse to medicate orthopedic postoperative patients for pain on an asneeded basis. Descriptive statistics were used to determine the participating nurses' demographics. The sample consisted of 404 nurses who were randomly chosen through their membership in the Association of Orthopedic Nurses (NAON). Participants completed a 57-item questionnaire. Of the 57 items, 17 were related to demographics, and 21 measured the participants' underlying beliefs about patient pain, what they believed were the outcomes of a behavior, what they believed the expectations were of important others concerning whether they should perform a behavior or not, and whether they perceived that they had control over the behavior. Other items pertained to the intentions of the participant to provide pain medication and the importance of the participants' behaviors. The other 11 questions were related to postoperative pain management.

The results showed that $98 \%$ of the participants planned to give opioids to their patients in the future. The percentage of nurses who reported that they intended to administer the maximum dose of as-needed opioids was $77.6 \%$. It was found that $32.4 \%$ 
of the nurse participants were most likely to give the as needed dose of opioids prior to the time allowed per orders between the doses. These results suggested that nurses continued to have concerns with administering opioids.

\section{Comfort Theory}

The nursing theory used to guide this project was Kolcaba's comfort theory. The comfort theory is a middle-range nursing theory developed in the 1990s (Petiprin, 2016). It states that comfort is the art of nursing (Petiprin, 2016). The comfort theory describes comfort as occurring in the forms of relief, ease, and transcendence (Petiprin, 2016).

This theory can be applied to many areas in health, particularly in relation to postoperative pain. Comfort can encompass physical, psychospiritual, sociocultural, and environmental factors (Petiprin, 2016). In the PACU, when a patient's pain is relieved, relief and ease occur. Transcendence is calm that occurs when an individual is overcoming challenges (Petiprin, 2016). The pain associated with surgery is a challenge, and relief of pain in the postoperative period allows for transcendence and the relief of anxiety related to this challenge.

Kolcaba defined the term patient as applying not only to individuals, but also to families, hospital units, hospitals, and even communities that seek improved health (Petiprin, 2016). The healthcare need identified is for an evidence-based pain management nursing protocol that will alleviate pain, allow for relief and ease of anxiety, and create transcendence by providing comfort for the postoperative patient. 


\section{Relevance to Nursing Practice}

There is a lack of knowledge of evidence-based pain management throughout many disciplines of healthcare, including nursing (Pasero et al., 2016). Nurses are at the forefront of providing evidence-based pain management. It is the bedside nurse who assesses the patient's pain level. It is the bedside nurse who provides medication to the patient. The lack of use of evidence-based pain management affects the patient's recovery time after surgery (Jungquist, et al., 2016). It impacts the hospital as an organization by potentially increasing patients' length of stay and healthcare expenditures while resulting in poor patient outcomes (Jungquist, et al., 2016). The most prevalent barrier to effective pain relief is lack of assessment (Jungquist, et al., 2016). If nurses are not effectively assessing patients for pain, then pain will not be treated effectively. In the United States and globally, postoperative pain is not managed effectively (Taylor et al., 2017). Although the opioid epidemic and opiophobia exist, nurses must continue to treat patients for their pain and must administer opiates (Manworren, Gordan, \& Montgomery, 2018). Opiates are an integral part of evidence-based pain management (Manworren et al., 2018). Annually, more than 50 million surgeries are performed in the United States. Of these surgical patients, more than $80 \%$ describe their postoperative pain as being moderate to severe and report inadequate pain relief (Manworren et al., 2018).

This doctoral project will fill the gap in practice involving the lack of an evidence-based pain management guideline in a PACU. The Centers for Medicare and Medicaid Services take the stance that all patients have the right to effective pain management and that they also have the right to participate in their plans of care (Harding 
\& Flynn-Harding, 2014). Pain management order sets are recommended by the ISMP (Pasero et al., 2016). Recommendations indicate that pain management order sets help to reduce the need to call providers for clarification of orders; these order sets should be clear, specific, and objective and should omit any range orders that are not attached to objective measures (Pasero et al., 2016). Inadequate evidence-based pain management education is a limitation among health care professionals (Pasero et al., 2016).

Evidence also suggests that the use of evidence-based pain management interventions for acute pain decreases the risk of progression to chronic pain (Pierik, et al., 2016). In addition to a knowledge deficit, lack of interdisciplinary communication, negative attitudes, and misconceptions among nursing staff contribute to ineffective pain management and the need for evidence-based pain management order sets and pain management protocols (Pierik, et al., 2016). Pain management protocols and order sets have been shown to not only decrease the time to administration of pain medicines, but also increase the number of patients who receive effective analgesia (Pierik, et al., 2016). Barriers to effective pain management among health care providers include fears of adverse effects of opioids and nurses not believing patients' own pain assessments (Drake \& Williams, 2017).

Research has repeatedly identified lack of knowledge among nurses as the most significant barrier to effective pain management (Mazilu, Zazu, Nedelcu, \& Sfetcu, 2018). Poor assessment of pain leads to poor patient outcomes, including deep vein thrombosis, pulmonary embolism, infections, inadequate healing of wounds, and cardiac issues (Mazilu et al., 2018). Research has supported recommendations for education, 
guidelines, and proper assessment tools for effective pain management (Chou et al., 2015).

Evidence based pain management guidelines have been developed and utilized in emergency departments with positive outocmes (Pierik, et al., 2016). Pain management guidelines and order sets have been shown to not only decrease the time to administration of pain medicines, but they also increase the number of patients who receive effective analgesia (Pierik, et al., 2016). Pain is undertreated in the PACU, and this ineffective pain management increases the post-surgical patient's risks of problems in the nervous system leading to chronic pain and related issues and an increased risk of a poorer quality

of life with unwarranted psychological suffering (Hayes \& Gordan, 2015). Evidence also suggests that the use of evidence-based pain management interventions for acute pain will decrease the risk of the progression to chronic pain (Pierik, et al., 2016).

\section{Local Background and Context}

This project took take place in a 15-bed PACU, in a 240-bed community hospital located in the Northeastern region of the United States, seeing approximately 9,000 patients annually. The PACU nursing staff consists of 26 registered nurses. All the nurses have a minimum education of a baccalaureate degree. They all have a minimum of 10 years' experience as registered nurses. Their experience includes intensive care, critical care, emergency nursing and multiple years of medical-surgical nursing experience. The chief of anesthesia, the director of pain management, 15 anesthesiologists, surgical NP's and PA's, CRNA's and the pain management nurse practitioner are the pain management experts available to the nurses in the PACU. 
There is the lack of an evidence-based pain management guideline in the PACU. The nurses express having difficulty differentiating between when a patient is sleeping versus experiencing unintended opioid sedation. Due to this phenomenon the PACU nurses' question whether to medicate a patient who complains of pain or to hold opioids. There is no use of an opioid sedation scale as recommended in the literature (Chou et al., 2015). Nurses are utilizing two pain assessment tools concurrently which may add to confusion as to which pain medication to provide. It has been noted through interviews that the nurses "feel compelled" to assess utilizing both scales due to the presence of both scales in the electronic medical record when utilizing analgesics and opioids. Often the nurses are utilizing the FLACC scale in patients who can speak for themselves as observed by the pain management nurse practitioner (Faron, 2018). A third practice noted is the lack of titration of analgesics and opioids. Per the pain management nurse practitioner, the nurses are unsure of when to give which medication and which dose (Faron, 2018). This project is supported by the chief of anesthesia, the director of pain management, the pain management nurse practitioner, management and the postanesthesia care unit nurses.

\section{Role of the DNP Student}

The role of the DNP is to be an expert in the translation of evidence into practice and to translate evidence into practice (White, Dudley-Brown, \& Terhaar, 2016). As the DNP student, I will be the facilitator and designer of the evidence-based pain management guideline and algorithm as guided by the evidence. I have experienced pain as a patient, witnessed it as a bedside nurse, and as a nurse practitioner. I am passionate 
about my patient's comfort. I have and continue to care for patients with postoperative pain, acute pain, chronic pain, cancer pain, and I have cared for patients who have a history of and actively suffering from substance use disorders. My motivation for completion of this project is to share the evidence on pain management to improve nursing care and patient outcomes. A lack of education on evidence-based pain management is a reality and through this project I hope to educate a group of nurses in a post anesthesia care unit in a community hospital to help dispel misconceptions about pain management.

\section{Summary}

The next section will describe the process for assembling an interdisciplinary team and the development and consensus process for an evidence-based nursing practice guideline in management of post-operative patient pain in the PACU. 
Section 3: Collection and Analysis of Evidence

\section{Introduction}

The practice problem addressed with this project is the lack of use of an evidencebased pain management guideline in the PACU. Nurses continue to practice through habit, pain management assessment tools are being used when not warranted, and nurses are having difficulty differentiating between sleeping and sedation and knowing when it is appropriate to medicate for pain. This project's goal was to create an evidence-based pain management guideline to help guide practice in the PACU. The role of the DNP is to translate current evidence into practice (White, Dudley-Brown, \& Terhaar, 2016). An

evidence-based pain management guideline, along with an algorithm for visual guidance, has been developed. Now that the guideline and algorithm have been developed, education on the use of these tools will commence. The expert panel involved in this project included an anesthesiologist, who is the director of pain management; a second anesthesiologist, who is the chief of anesthesia; a surgical NP, who practices as a surgical first assist and sees patients in the postoperative care unit.

\section{Practice-Focused Question(s)}

In this community hospital, an evidence-based pain management guideline is lacking in the PACU. The gap in practice results in the nurses continue to practice out of habit, having difficulty differentiating between sleeping and sedation, using more than one pain assessment tool concurrently, and feeling unsure of when it is appropriate to medicate for pain. The nurses are not provided with the tools they need to provide the best care to their patients. They have personally expressed these concerns. The practice 
focused question is the following: Can an interdisciplinary team develop an evidencebased pain management guideline to help improve pain management in PACU patients? The gap in practice will be addressed through the developed evidence-based pain management guideline for use in the PACU in a community hospital.

The purpose of this project was to develop an evidence-based pain management guideline for the PACU of a community hospital to improve patient outcomes and nursing practice. Evidence supports the titration of analgesics according to their effect and adverse outcomes (Ballantyne et al., 2016). It is recommended that nurses use an opiate sedation scale when administering opioids (Pasero et al., 2016). Evidence-based pain management guidelines have been shown to improve patient outcomes (Barksdale et al., 2016). Therefore, the implementation of an evidence-based pain management guideline that includes the proper titration of analgesics and an opioid sedation scale, along with the NPS, should improve patient outcomes.

\section{Sources of Evidence}

Evidence was collected via multiple databases. These included CINAHL, Medline, Science Direct, ProQuest health, Medical Collection, Allied Health Source, Cochrane and PubMed. These search engines were located within the Walden Library. The search frame included the years from 2012 to the present. The evidence included peer-reviewed journal articles, research studies, and guidelines. Search terms included pain, pain management, post-anesthesia care unit, postoperative pain, pain assessment, pain assessment tools, opioids, opiates, opioid adverse effects, opioid titration, 
analgesics, WHO analgesics, pain management nursing, comfort, nursing theories, and palliative care.

The evidence collected, the Comfort theory, and the Integrated Behavioral Model were used as guides. The guideline was developed along with an algorithm for visual guidance. The team providing input and evaluation of the guidelines included the chief of anesthesia, the director of pain management, the pain management NP, a surgical NP, and an anesthesiologist.

\section{Summary}

An evidence-based pain management guideline is lacking in the PACU in this community hospital. The PACU nurses are not provided with an opioid sedation scale as recommended. Analgesics and opioids are not being titrated based on effect and adverse reactions. The postoperative orders must include the ability for the nurses to titrate analgesics and opioids based on their assessment findings. An order set will be created to include the ability to properly titrate opiates based on the assessment findings of the nurse and reflect these guidelines. The nurses are also using more than one pain assessment tool concurrently, including the NPS and the FLACC scale. Pain management guidelines have been shown to improve outcomes. Effective acute pain management decreases the risks

of suffering from chronic pain, decreases length of stay, improves patient satisfaction and decreases morbidity and mortality. This project will translate evidence into practice and will improve nursing practice and patient outcomes. In Section 4, I describe the process for developing the guidelines. 
Section 4: Findings and Recommendations

\section{Introduction}

An evidence-based pain management guideline is lacking in the PACU in this acute care facility. This quality improvement project involved the development of a clinical practice guideline for use in the PACU. This clinical practice guideline includes an algorithm for visual guidance for the nurses. It includes both the NPS and the POSS. The purpose of this project was to provide this PACU with an evidence-based pain management guideline and provide nurses with the tools to guide their interventions and improve patient outcomes.

\section{Key Sources of Evidence}

The guidelines were developed using APS and National Comprehensive Cancer Network (NCCN) guidelines for evidence-based pain management in the PACU. The APS has provided guidelines for the management of postoperative pain. The NCCN has published guidelines for adult cancer pain. The NCCN guidelines include assessment of pain, management of pain in the opiate-naive patient, pain management during a pain crisis, and opioid prescribing, titration, and maintenance for adult cancer patients. A pain crisis is pain that is unrelieved by an individual's current pain management regimen.

Professional organizations' guidelines that were used in developing this guideline included the WHO analgesic ladder (WHO, 2018), the APS 2015 Post-Operative Pain Management Guidelines (Chou et al., 2015), the American Society of Anesthesiologists 2015 Post-Operative Pain Management Guidelines (Chou et al., 2015), the National Hospice and Palliative Care Organization's guidelines for the titration of opioids 
(National Hospice and Palliative Care Organization, 2018), and the NCCN guidelines (NCCN, 2018).

Chou et al. (2015) developed a clinical practice guideline for evidence-based pain management. Of the 32 recommendations within that guideline, the following three were most relevant to this project:

- Recommendation 3, that a preoperative evaluation be performed to identify a history of chronic pain, substance use disorder, and previous pain management interventions that were effective.

- Recommendation 4 to adjust the pain management plan according to effect and adverse reactions.

- Recommendation 5 suggesting the use of assessment and reassessment of pain management interventions, including assessment of pain and sedation (Level of Evidence I).

A study by Barksdale et al. (2016) showed that the use of an emergency department pain management guideline decreased the time between a patient's complaint of pain and the administration of pain medications (Level of Evidence IV).

\section{Findings and Implications}

This guideline is based on evidence-based assessment tools for use in pain management, proper titration of opiates, and guidance in notifying a specialist if pain management has been ineffective. It also provides guidance with proper opiate conversions using the equianalgesic chart (Appendix D) with morphine as the gold 
standard. This guideline is an adaptation of the guidelines provided by the WHO, the NCCN, and the studies reviewed for the guideline's development.

This guideline presents nurses and other providers with the NPS, which is accepted as an evidence-based pain assessment tool. It also includes the POSS, which has been supported by evidence and recommended by the Joint Commission for use when an opiate is administered. The guideline provides users with recommended starting doses for mild, moderate, and severe pain. It provides a decision tree or algorithm for users to follow when pain is unrelieved (Appendix B). The strength of the evidence ranges from Level 1 to Level 5 as assessed by the development team. A hierarchy of evidence for intervention studies adapted from the levels of evidence presented by Sackett (1996) and the Grading of Recommendations, Assessment, Development, and Evaluation (GRADE) approach (Zhang et al., 2017) were used to evaluate the strength of the evidence. The five levels of strength included in the hierarchy of evidence for intervention studies are as follows:

Level 1: Systemized review and randomized control studies

Level 2: Cohort studies

Level 3: Case-control studies

Level 4: Case reports

Level 5: Narrative review studies

The key recommendations that came from the literature review are provided below. 


\section{Disease/Condition}

The disease/condition is postoperative pain.

\section{Guideline Category}

The guideline corresponds with the following categories: pain assessment, sedation assessment, prevention of chronic pain and unwarranted acute pain; and treatment of acute pain and acute/chronic pain.

\section{Adequate Pre- and Postoperative Assessment—Criteria}

Recommendation 1. The NPS is to be used on all patients who can speak and can count to 10 (Pasero et al., 2016). This includes sleeping patients. It is recommended to awaken a patient for reassessment to assess for pain management effectiveness and sedation (Davis, et al., 2017). Always awaken patient and reassess prior to transfer out of

PACU. Assess patient every 15 minutes after administering opioid and/or nonopioid medication.

The FLACC scale is recommended for use in pediatric patients who are unable to use the NPS. It is not to be used alongside the NPS or if a patient is able to speak and count to 10 .

Recommendation 2. The POSS is to be used on all patients who are receiving opioids. This scale ranges from 1 to 4 and is to be used at the same time as the NPS or the FLACC scale (Glowacki, 2015). 


\section{Recommendations for Titration}

Recommendation 3. Patients with a history of chronic pain and the use of opioids should continue their at-home regimens throughout the perioperative period (Chou et al., 2015; Pasero et al., 2016).

Recommendation 4. Patients with a history of chronic pain and use of opioids may require higher doses than the recommended doses due to tolerance, and therefore an expert consult is recommended (NCCN, 2018). The titration recommendation is to increase or decrease doses by $50-100 \%$ based on effect and adverse effects (NCCN, 2018).

Recommendation 5. For treatment of mild pain, it is recommended to use a nonopioid or weak or low-dose opioid, either orally or through the IV as first-line treatment in the PACU (WHO, 2018).

The titration recommendation is that if the initial dose is ineffective, that dose should be increased by $50-100 \%$ after approximately 15 minutes. Opioids have different times to onset and peak of action, which are documented in the opioid equianalgesic chart (Appendix D). If the opioid has not been effective at peak effect, it will not be effective, and titration is necessary.

Recommendation 6. It is recommended to avoid administering benzodiazepines in congruence with opiates. Common benzodiazepines include Valium, Ativan, and Versed. Benzodiazepines should not be given to patients until pain is under control and should only be used in extreme cases with severe spasms. 
Recommendation 7. If adverse effects occur including sedation, such as nausea and vomiting, and the patient continues to report pain, it is recommended to decrease the last administered dose by $50 \%$ or to hold medications until the adverse effects improve (NCCN, 2018). Antiemetics should be included in the pain management order sets along with narcan for oversedation.

Recommendation 8. For the treatment of moderate pain, a nonopioid plus a moderate-dose opioid should be first-line treatment (WHO, 2018). Again, the patient's status of opiate naïve or tolerant must always be considered prior to ordering the proper dose. Please use the opioid equianalgesic chart for reference. If this dose is ineffective, increase dose by 50-100\%. If adverse effects occur and patient continues to report pain, decrease dose by $50 \%$

Recommendation 9. It is recommended to create order sets specifically for the PACU as guided by this protocol. The order sets should include the following:

- One medication for each level of pain

- One medication for oral and one for IV, preferably the same medication

- One duration

- One route $\mathrm{PO} / \mathrm{IV}$

Recommendation 10. It is recommended to follow the algorithm to include treatment at the second assessment by increasing the opiate dose by $50 \%$ if the first dose is ineffective after 15 minutes, and at the third assessment by increasing the dose by $50 \%$ if the second dose is ineffective. If pain continues, it is recommended to seek expert consultation. 
Recommendation 11. It is recommended to provide discharge criteria that must be met prior to transferring the patient to the inpatient unit or the ambulatory surgery unit. If these criteria are not met, then the patient should continue to have intensive monitoring. Discharge criteria should include at least the following: a pain score approximately $30 \%$ lower than the initial pain score, increase in function and/or patient expressing satisfaction with present pain intensity, and a POSS of 1 or 2.

Recommendation 12. A guideline should be provided for patient monitoring on the inpatient and ambulatory surgery units to include the continued use of the NPS and POSS.

Recommendation 13. If a patient experiences a POSS of 3, then it is recommended to notify an anesthesiologist, the pain management team, or any provider available.

If oversedation or a POSS of 4 occurs, it is recommended to call anesthesia, protect the airway, and provide Narcan slowly as per hospital protocol.

\section{Clinical Specialty}

The clinical specialty is surgery.

\section{Intended Users}

Intended users of the guideline include advanced practice nurses, physician assistants (PAs), hospitalists, surgeons, anesthesiologists, PACU RNs, Surgical NPs, Surgical PA's and CRNA's (certified registered nurse anesthetists). 


\section{Outcomes}

Expected outcomes of guideline implementation include decreased numeric pain scores, shorter interval between reports of pain and treatment of pain, proper titration of opiates to decrease pain and risk of adverse effects, assessment of sedation (which precedes respiratory adverse reactions), decreased morbidity and mortality, decreased risk of suffering from chronic pain, decreased surgical infections, improved patient satisfaction and improved outcomes, and decreased need to escalate issues to experts.

\section{Contribution of the Project Team}

An expert panel was assembled for review of the final draft of the guideline (Appendix E). This panel included the medical director of pain management, the chief of anesthesia, and the associate director of robotic surgery, who is an NP surgical first assistant.

The purpose of this project was to develop an evidence-based pain management clinical practice guideline to be used in the PACU. The expert panel reviewed the clinical practice guidelines using the Agree II tool. Domains 1 through 6 were reviewed and applied to the tool. Under each domain, all members of the team strongly agreed with the recommendations. One member was unclear on the activities of the project team. I developed the guideline and used the members of the team as the expert panel. Once this was explained, the panel members expressed full understanding. This project was fully supported by the three members of the panel. One member needed assurance that the bedside nurses would be fully involved and educated. This will occur during the 
dissemination of the project. The nurse manager of the PACU and the PACU nurse flow coordinator have reviewed the guidelines and fully support the project.

Maximum possible score $=7$ (strongly agree) $\times 3$ (items) $\times 3$ (appraisers) $=63$

Minimum possible score $=1$ (strongly disagree) $\times 3$ (items) $\times 3$ (appraisers) $=9$

The scaled domain score will be:

Obtained score - Minimum possible score

Maximum possible score - Minimum possible score

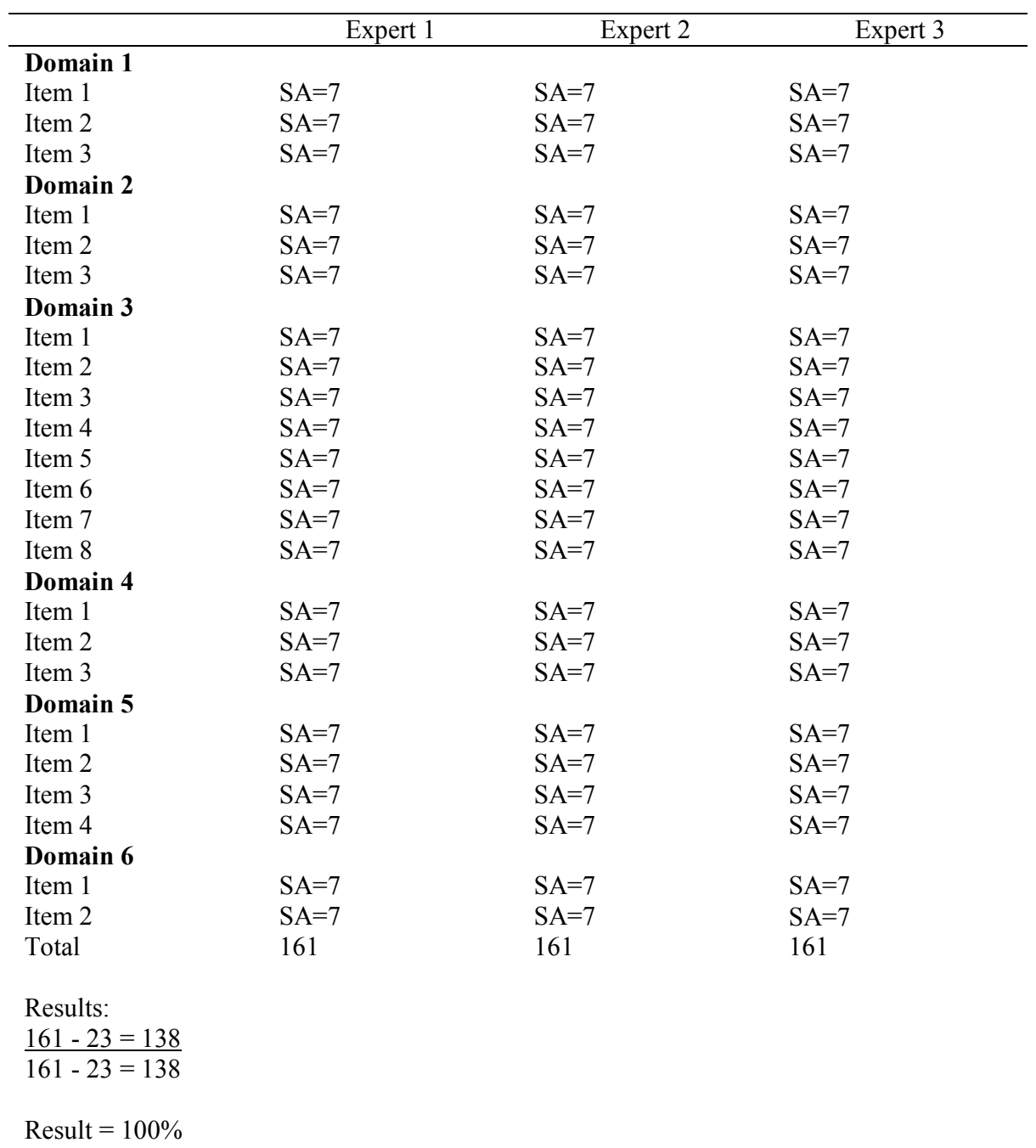

Results: "High quality guidelines are those with domain scores that are all $>70 \%$ " (Brouwers, 2017, p. xx). The guidelines have domain scores greater than $70 \%$; therefore, they are high-quality guidelines.

Figure 1. Results of the Agree tool provided by the expert panel. 


\section{Recommendations}

I recommend that the PACU nurses use the developed evidence-based pain management guideline and the algorithm developed to visually assist its use. I recommend that the guideline move forward for review by specific approvals required by the facility, that the nurses be adequately educated, and that the results be monitored for 6-12 months to determine the effectiveness of the guideline. Education on the use of the guideline is necessary prior to its use, and that knowledge should be evaluated annually. Education must address the need to overcome myths and historical apprehensiveness when providing opiates, particularly in relation to concerns that have been fueled by the opioid crisis. All staff nurses and members of management will need to be educated on the use of the guideline, and future confidence and comfort among the guideline's users should be monitored and evaluated.

The effectiveness of the guideline should be measured at 3, 6, and 12 months after implementation for nurse satisfaction, patient pain relief scores correlated with use of medications/ dosing, and requests for intervention from the NP and anesthesiologists. If the use of this guideline achieves the desired outcome, it should be considered for use throughout the hospital for improved patient outcomes.

\section{Strengths and Limitations of the Project}

The strengths of this project include the support from the administrators of the hospital where this project took place. The expert panel fully supports the created guideline and will be integral to its successful implementation. Financial support has been provided for education processes needed for implementation. Full support has been 
provided by the nursing education and quality departments for implementation of these guidelines. Limitations include limited studies available on the use of titration of opiates in the PACU setting and acute pain management.

After gathering the expert panel to evaluate the project utilizing the Agree tool, I found that the project recommendations were fully supported by all members of the panel. The strengths include the support of the expert panel, including the nurse manager of the PACU and the PACU flow coordinator. Support from administration and nursing education is an important aspect of the project that will support dissemination of the protocol. The PACU nurses provided support for the project and expressed the continued need for the implementation of the recommendations. 
Section 5: Dissemination Plan

The plan for dissemination will begin with the introduction of the completed evidence-based clinical practice guideline to the PACU nurses and providers who will be using it. This process will begin with a PowerPoint educational program that will be presented repeatedly over one full week. I will present the guideline each day in the PACU until all users are fully aware of the guideline. Next, the guideline will be presented to the evidence-based council and the informatics council.

In the following week, I will meet with the medical chief of informatics to assure that the medical order set for the PACU is in place for providers to properly order the medications in alignment with the guideline. Once the orders are in place, I will educate the providers, including anesthesiologists, NPs, PAs, and surgeons, on the location of the orders and the content of the order set. I will educate the pharmacists on the guideline and order set once it is $\mathrm{n}$ the medical information system.

Once the order set has been completed and is live in the medical information system, I will assist providers in the ordering process by being available at the bedside when a patient arrives in the PACU. I will also be available at the bedside for the PACU nurses to assist in the guideline's use. I will have champions, including the nurse flow coordinator, the $\mathrm{RN}$ who is a member of the evidence-based council, and the nurse educator for perioperative services, assist with education on the guideline and its use at the bedside.

This project could be presented to the board of trustees, the board of medicine, the nursing leadership council, and in nursing grand rounds. In the future, this project could 
also be presented at the peri-anesthesia nursing conference. This project could be adapted for application in all hospital areas, including the medical units and the emergency department.

I have learned a lot about myself through this journey. Through this process, I have learned about the proper use of opiates and how to properly, safely, and effectively manage pain. I have learned about evidence-based assessment tools and what is lacking in education about proper pain management. I was unaware of the complex nature of pain management, and I am excited to educate others for the benefit of patients. I am excited to present the findings of the project and want to help bring together nursing and medicine through education. This project shows evidence that those in nursing and medicine must work together and that we are dependent on one another to provide safe and effective patient care. Using guidelines, evidence can be disseminated and integrated into patient care, leading to improved patient outcomes.

Through this project, I have gained confidence, knowledge, and comfort as a practitioner. In the future, I would love to become an educator. I recently was invited to speak at an advanced illness conference on evidence-based pain management. I have also been asked to chair a leadership pain management task force. I am currently a member of a system-wide pain management task force.

I hope that the completion of this project will occur in the next 2 months. I will experience challenges. Challenges that I have faced mirror the evidence mentioned in this project. I have met and continue to meet challenges that result from the lack of knowledge that exists nationally among healthcare professionals on evidence-based pain 
management. I recognize the fear of opiates and the misconceptions about the use of opiates that continue to exist among both nursing and medical providers. The best way to overcome lack of education, fears, and misconceptions is to be an active educator and to share my knowledge. I believe that supporting nurses at the bedside- being mindful not to be judgmental but encouraging and understanding — will be the best way to help them build their knowledge and gain confidence and comfort in the management of pain in the PACU. I hope that this project will be met with positivity.

\section{Summary}

The Centers for Medicare and Medicaid Services take the stance that all patients have the right to effective pain management and that they also have the right to participate in their plans of care (Harding \& Flynn-Harding, 2014). Pain management order sets are recommended by the ISMP (Pasero et al., 2016). It has been noted that pain management order sets help to reduce the need for nurses to call providers for clarification of orders. Order sets should be clear, specific, and objective and should omit any range orders that are not attached to objective measures (Pasero et al., 2016). Inadequate evidence-based pain management education is a limitation among health care professionals (Pasero et al., 2016).

Evidence also suggests that the use of evidence-based pain management interventions for acute pain will decrease the risk of progression to chronic pain (Pierik et al., 2016). In addition to a knowledge deficit in nursing, lack of interdisciplinary communication, negative attitudes, and misconceptions contribute to ineffective pain management and the need for evidence-based pain management order sets and pain 
management guidelines (Pierik, et al., 2016). Pain management guidelines and order sets have been shown to not only decrease the time to administration of pain medicines, but also increase the number of patients who receive effective analgesia (Pierik, et al., 2016). Barriers to effective pain management among health care providers include fears of adverse effects of opioids and nurses not believing patients' own pain assessments (Drake \& Williams, 2017).

There have been repeated reports indicating that lack of knowledge among nurses is the most significant barrier to effective pain management (Mazilu et al., 2018). Poor assessment of pain leads to poor patient outcomes, including deep vein thrombosis, pulmonary embolism, infections, inadequate healing of wounds, and cardiac issues (Mazilu et al., 2018). The research supports recommendations for education, guidelines, and proper assessment tools for effective pain management (Chou et al., 2015).

Evidence-based pain management guidelines have been developed and used in emergency departments with positive outocmes (Pierik, et al., 2016). Pain management guidelines and order sets have been shown to not only decrease the time to administration of pain medicines, but also increase the number of patients who receive effective analgesia (Pierik, et al., 2016). Barriers to effective pain management among health care providers include fears of adverse effects of opioids and nurses not believing patients' own pain assessments (Drake \& Williams, 2017). Pain is undertreated in the PACU, and ineffective pain management increases post-surgical patients' risks of problems in the nervous system leading to chronic pain and related issues as well as increased risk of poorer quality of life with unwarranted psychological suffering (Hayes \& Gordan, 2015). 
Evidence also suggests that the use of evidence-based pain management interventions for acute pain will decrease the risk of the progression to chronic pain (Pierik, et al., 2016). In addition to a knowledge deficit, lack of interdisciplinary communication, negative attitudes, and misconceptions contribute to ineffective pain management and the need for evidence-based pain management order sets and pain management guidelines (Pierik, et al., 2016).

The use of an evidence-based pain management guideline in the PACU will improve patient outcomes. It will improve nurses' and providers' knowledge of pain management. It will provide comfort and confidence in providing safe and effective pain management in the PACU. 
References

AGREE Next Steps Consortium. (2017, Aug 30). Retrieved from The Agree II instrument: http://www.agreetrust.org.

Ballantyne, J., Kalso, E. K., \& Stannard, C. (2016). WHO analgesic ladder: A good concept gone astray. British Medical Journal, 352.

Barksdale, A., Hackman, J., Williams, K., \& Gratton, M. (2016). ED triage pain protocol reduces time to receiving analgesics in patients with painful conditions. American journal of emergency medicine, 34, 2362-2366.

Centers for Disease Control and Prevention. (2015, June). Applying the Knowledge-toAction (K2A) framework: Questions to guide planning. Retrieved from https://www.cdc.gov/chronicdisease/pdf/k2a-framework-6-2015.pdf

Chou, R., Gordan, D., De Leon-Casasola, O., Rosenberg, J., \& Bickler, S. (2015). Guidelines on the management of postoperative pain. The Journal of pain, $x x$, $131-146$.

Current Nursing. (2011, February 10). Comfort theory: Katharine Kolcaba. Retrieved from http://currentnursing.com/nursing_theory/comfort_theory_Kathy _Kolcaba.html

Davis, C., Geik, C., Arthur, K., Fuller, J., Johnston, E., Levitt, F., . . Walroth, T. (2017). A multisite retrospective study evaluating the implementation of the Pasero Opioid-Induced Sedation Scale (POSS) and its effect on patient safety outcomes. Pain management nursing, 18, 193-201. 
Douma, M., Drake, C., O'Dochartaigh, D., \& Smith, K. (2016). A Pragmatic Randomized Evaluation of a Nurse-Initiated Protocol to Improve Timeliness of Care in an Urban Emergency Department. Annals of emergency medicine, 68 , 5 , 546 - 552.

Faron, S. (2018, January 15). Pain management nurse practitioner. (S. Mogan, Interviewer)

Jungquist, C., Correll, D., Fleisher, L., Gross, J., Gupta, R., Pasero, C., . . Polomano, R. (2016). Avoiding adverse events secondary to opioid-induced respiratory depression. Journal of nursing administration, 46, 87-94.

Kobelt, P., Burke, K., \& Renker, P. (2014). Evaluation of a standardized assessment for opioid administration in the post anesthesia care unit. Pain management nursing, $15,672-81$.

Manworren, R., Gordan, D., \& Montgomery, R. (2018). Managing postoperative pain. American journal of nursing, 118, 36-43.

Mazilu, D., Zazu, M., Nedelcu, V., \& Sfetcu, R. (2018). Effectiveness of pain management educational interventions on nurses' knowledge and attitudes regarding postoperative pain management: a systematic review protocol. Joanna Briggs Institute Database of Systematic Reviews and Implementation Reports, $16(2), 303-307$.

Mcpherson, M. (2010). Demystifying opioid conversion calculations: A guide for effective dosing. American society of health systems pharmacy.

Pasero, C., Quinlan-Colwell, A., Rae, D., Broglio, K., \& Drew, D. (2016). American Society for Pain Management Nursing position statement: Prescribing and administering opioid doses based solely on pain intensity. Pain Management 
Nursing, 17, 170-180.

Petriprin, A. (2016). Kolcaba's theory of comfort. Retrieved from http://www.nursingtheory.org/theories-and-models/kolcaba-theory-of-comfort.php

Pierik, J., Berben, S., Ijzerman, M., Gaakeer, M., Eenennaam, F., \& Van Vugt, A. (2016). A nurse-initiated pain protocol in the ED improves pain treatment in patients with acute musculoskeletal pain. International emergency nursing, 27, 3-10.

Reville, B., \& Foxwell, A. M. (2014). The global state of palliative care-Progress and challenges in cancer care. Annals of palliative medicine, 3(3), 129-138.

Ridderikhof, M., Schyns, F., Schep, N., Lirk, P., Hollman, M., \& Goslings, C. (2017). Emergency Department Pain Management in Adult Patients With Traumatic Injuries Before and After Implementation of a Nurse-Initiated Pain Treatment Protocol Utilizing Fentanyl for Severe Pain. The journal of emergency medicine, $52,4,417-425$.

Schroeder, D., Hoffman, L., Fioravanti, M., Medley, D., Zullo, T., \& Tuite, P. (2016). Enhancing nurses' pain assessment to improve patient satisfaction. Orthopaedic nursing, 35, 108-117.

Taylor, C., Sheu, J., Chen, H., Glassman, T., \& Dake, J. (2017). Predictors of nurses’ intentions to administer as-needed opioid analgesics for pain relief to postoperative orthopaedic patients in the acute care setting. Orthopaedic nursing, 36, 392-399.

White, K., Dudley-Brown, S., \& Terhaar, M. (2016). Translation of evidence into nursing and health care. New York, NY: Springer. 
World Health Organization. (2018). Sexual and reproductive health: Knowledge-toAction (KTA) framework. Retrieved from http://www.who.int/reproductivehealth /topics/best_practices/greatproject_KTAframework/en/ 
Appendix A: Agree Tool (AGREE Next Steps Consortium, 2017)

\section{AGREE II RATING OF CLINICAL PRACTICE GUIDELINES Rating Scale: (1) Strongly Agree, (2) Agree, (3) Partially Agree, (4) Neutral, (5) Partially Disagree, (6) Disagree, (7) Strongly Disagree}

\begin{tabular}{|c|c|c|c|c|c|c|c|}
\hline Domain & SA & $\mathbf{A}$ & PA & $\mathbf{N}$ & PD & D & SD \\
\hline The overall objective of the guideline is specifically described & & & & & & & \\
\hline $\begin{array}{l}\text { 2. The health question covered by the guideline is specifically } \\
\text { described }\end{array}$ & & & & & & & \\
\hline $\begin{array}{l}\text { 3. The population to whom the guideline is meant to apply is } \\
\text { specifically described }\end{array}$ & & & & & & & \\
\hline $\begin{array}{l}\text { 4. The guideline development group includes individuals from all } \\
\text { the relevant professional groups }\end{array}$ & & & & & & & \\
\hline $\begin{array}{l}\text { 5. The views and preferences of the target population have been } \\
\text { sought }\end{array}$ & & & & & & & \\
\hline 6. The target uses of the guidelines are clearly defined & & & & & & & \\
\hline 7. $\quad$ Systematic methods were used to search for evidence & & & & & & & \\
\hline 8. The criteria for selecting the evidence are clearly described & & & & & & & \\
\hline $\begin{array}{l}\text { 9. The strengths and limitations of the body of evidence are } \\
\text { clearly described }\end{array}$ & & & & & & & \\
\hline $\begin{array}{l}\text { 10. The methods for formulating the recommendations are clearly } \\
\text { described }\end{array}$ & & & & & & & \\
\hline $\begin{array}{l}\text { 11. The health benefits, side effects and risks have been } \\
\text { considered in formulating the recommendations }\end{array}$ & & & & & & & \\
\hline $\begin{array}{l}\text { 12. There is an explicit link between the recommendations and } \\
\text { the supporting evidence }\end{array}$ & & & & & & & \\
\hline $\begin{array}{l}\text { 13. The guideline has been externally reviewed by experts prior to } \\
\text { its publication }\end{array}$ & & & & & & & \\
\hline 14. A procedure for updating the guideline is provided & & & & & & & \\
\hline 15. The recommendations are specific and unambiguous & & & & & & & \\
\hline $\begin{array}{l}\text { 16. The different options for the management of the condition or } \\
\text { health issue are clearly presented }\end{array}$ & & & & & & & \\
\hline 17. Key recommendations are easily identifiable & & & & & & & \\
\hline $\begin{array}{l}\text { 18. The guidelines provide advice and/ or tools on how the } \\
\text { recommendations can be put into practice }\end{array}$ & & & & & & & \\
\hline $\begin{array}{l}\text { 19. The guidelines describe facilitators and barriers to its } \\
\text { application }\end{array}$ & & & & & & & \\
\hline $\begin{array}{l}\text { 20. The potential resource implications of applying the } \\
\text { recommendations has been considered }\end{array}$ & & & & & & & \\
\hline 21. The guidelines present monitoring and/ or auditing criteria & & & & & & & \\
\hline $\begin{array}{l}\text { 22. The views of the funding body have not influenced the content } \\
\text { of the guideline }\end{array}$ & & & & & & & \\
\hline $\begin{array}{l}\text { 23. Competing interests of guideline development group } \\
\text { members have been recorded and addressed }\end{array}$ & & & & & & & \\
\hline
\end{tabular}

\section{Comments:}


Appendix B: Mogan Evidence-Based Pain Management Algorithm

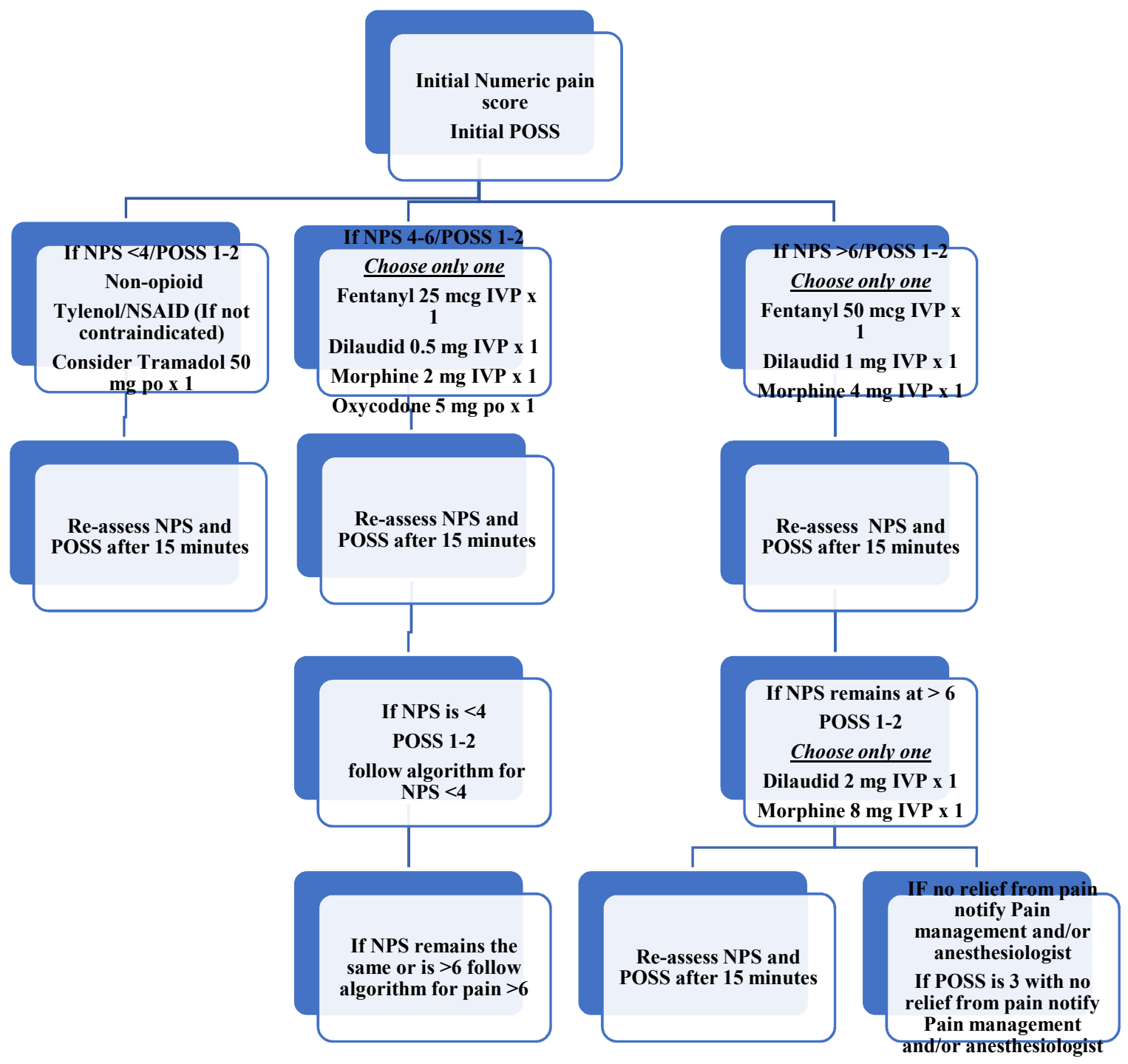


Appendix C: Pasero Opioid-Induced Sedation Scale (POSS) (Davis, et al., 2017)

\section{Pasero Opioid-Induced Sedation Scale (POSS)}

$\mathbf{S}=$ Sleep, easy to arouse

$\mathbf{1}=$ Awake and alert; acceptable may administer opiate

$\mathbf{2}=$ Slightly Drowsy, easily aroused; Acceptable, may administer opiate

$\mathbf{3}=$ Frequently drowsy, arousable, drifts off during conversation

Do not administer opiate

$4=$ Somnolent, minimal to no response to physical stimulation

Unacceptable; do not administer opiate

Consider capnography

Arrives to PACU

Numeric pain assessment initial 0-10

POSS score 1-4

- $\quad$ NPS $<$ or equal to 4, POSS 1 or 2, non-opiate analgesia, Tylenol/NSAID

- NPS 4-6, POSS 1-2, non-opiate and offer a mild opiate, oxycodone $5 \mathrm{mg}$ po, tramadol 50 $\mathrm{mg}$ po, dilaudid $0.5 \mathrm{mg}$ IVP, Fentanyl $25 \mathrm{mcg}$ IVP, Morphine $2 \mathrm{mg}$ IVP

- NPS> 6, POSS 1 or 2, increase opiate, oxycodone $10 \mathrm{mg}$ po, Dilaudid $1 \mathrm{mg} I \mathrm{IV}$, Morphine $6 \mathrm{mg}$ IVP

- Re-assessment after 15 minutes;

- NPS continues to be > 6;

- Increase the opiate by $50-100 \%$

If pain score remains above 6 or POSS is 3-4 notify anesthesia or pain management $\mathrm{NP} / \mathrm{MD}$

We are looking to relieve pain by approx. $30 \%$

May use Dilaudid po in place of oxycodone if patient cannot tolerate oxycodone

Dilaudid $2 \mathrm{mg}$ po for oxycodone $5 \mathrm{mg}$ po, Dilaudid $4 \mathrm{mg}$ po for oxycodone $10 \mathrm{mg}$ po 
Appendix D: Opioid Equianalgesic Chart (Mcpherson, 2010)

\section{Equianalgesic Opioid Dosing}

\begin{tabular}{lcc} 
& \multicolumn{2}{c}{ Equianalgesic Doses $(\mathbf{m g})$} \\
\cline { 2 - 3 } Drug & Parenteral & Oral \\
\hline Morphine & 10 & 30 \\
\hline Buprenorphine & 0.3 & $0.4(\mathrm{sl})$ \\
\hline Codeine & 100 & 200 \\
\hline Fentanyl & 0.1 & NA \\
\hline Hydrocodone & NA & 30 \\
\hline Hydromorphone & 1.5 & 7.5 \\
\hline Meperidine & 100 & 300 \\
\hline Oxycodone & $10^{\star}$ & 20 \\
\hline Oxymorphone & 1 & 10 \\
\hline Tramadol & $100^{\star}$ & 120 \\
\hline
\end{tabular}

McPherson ML. Demystifying Opioid Conversion Calculations: A Guide For Effective Dosing. Amer 
Appendix E: Evidence-Based Pain Management Guidelines for the Postanesthesia Care Unit

Disease/Condition: Post-operative pain

Guideline Category: Pain assessment, Sedation Assessment, Prevention of chronic pain and unwarranted acute pain; Treatment of acute pain and acute/chronic pain.

\section{Adequate pre and post-operative assessment - criteria}

\section{Recommendation 1:}

The Numeric Pain Scale or NPS is to be used on all patients who can speak and can count to 10 (Pasero, Quinlan, Rae, \& Broglio, 2016). This includes sleeping patients. It is recommended to awake a patient for reassessment to assess for pain management effectiveness and sedation (Pasero, Quinlan, Rae, \& Broglio, 2016). Always awaken patient and reassess prior to transfer out of PACU. Assess patient q15minutes after administering opioid and/or non-opioid.

The FLACC scale: Faces, Legs, activity, crying, consolability is recommended for use in pediatric patients who are unable to utilize the Numeric pain Scale. It is not to be used alongside the NPS or if a patient is able to speak and count to ten (Pasero, Quinlan, Rae, \& Broglio, 2016).

\section{Recommendation 2:}

The Pasero Opioid Sedation Scale or POSS is to be used on all patients who are receiving opioids (Joint commission, 2018). This scale is based 
on a scale from 1 to 4 and is to be used at the same time as the NPS or the FLACC scale (Glowacki, 2015).

\section{Proper use of orders for titration of pain medications based on guideline.}

\section{Recommendations for titration:}

\section{Recommendation 3:}

Patient's with a history of chronic pain and the use of opioids should continue their at home regimen throughout the perioperative period (Chou, Gordan, De Leon-Casasola, Rosenberg, \& Bickler, 2015) (Pasero, Quinlan, Rae, \& Broglio, 2016)

\section{Recommendation 4:}

Patients with a history of chronic pain and use of opioids may require higher than the recommended doses due to tolerance and therefore an expert consult is recommended (Chou, Gordan, De Leon-Casasola, Rosenberg, \& Bickler, 2015); Titration recommendations of increasing or decreasing doses by $50-100 \%$ based on effect and adverse effects are the same for this population (NCCN, 2018).

\section{Recommendation 5:}

For treatment of mild pain, it is recommended to utilize a non-opioid or weak or low dose opioid po/IV as first-line treatment in the PACU (The world health organization, 2018).

If initial dose is ineffective increase that dose by $50-100 \%$ after approximately 15 minutes (NCCN, 2018). Each opioid has a different time 
to onset and peak of action which are documented in the opioid equianalgesic chart (Appendix D). If the opioid has not been effective at peak effect it will not be effective, and titration is necessary (NCCN, 2018).

\section{Recommendation 6:}

It is recommended to avoid administering benzodiazepines in congruence with opiates. The benzodiazepines include; Valium, Ativan and Versed. Benzodiazepines should not be given to patients until pain is under control and should only be used in extreme cases with severe spasms.

\section{Recommendation 7:}

If adverse effects occur including sedation, nausea and vomiting and the patient continues to report pain it is recommended to decrease the last administered dose by $50 \%$ or to hold medications until the adverse effects improve. Antiemetics should be included in the pain management order sets along with Narcan for over-sedation.

\section{Recommendation 8:}

For the treatment of moderate pain, a non-opioid plus a moderate dose opioid should be first-line treatment. Again, the patient's status of opiate naïve or tolerant must always be considered prior to ordering the proper dose. Please utilize the opioid equianalgesic chart for reference (Appendix D). If this dose is ineffective increase dose by $50-100 \%$. If adverse effects occur and patient continues to report pain decrease dose by $50 \%$ 
Recommendations 9:

It is recommended to create order sets specific for the PACU as guided by this protocol the order sets should include:

One medication for each level of pain

One medication for oral and one for IV preferably the same medication

One duration

One route po/IV

\section{Recommendation 10:}

It is recommended to follow the algorithm to include treatment at second assessment by increasing opiate dose by $50 \%$ if first dose ineffective after 15 minutes and at third assessment increase dose by $50 \%$ if second dose is ineffective. If pain continues it is recommended to seek expert consultation.

\section{Recommendation 11:}

It is recommended to provide discharge criteria that must be met prior to transferring the patient to the inpatient unit or the ambulatory surgery unit. If these criteria are not met, then the patient should continue to have intensive monitoring. Discharge criteria should include at least the following; a pain score approximately $30 \%$ lower than the initial pain 
score, increase in function and/or patient expressing satisfaction with present pain intensity and a POSS of 1 or 2 .

\section{Recommendation 12:}

This guideline recommends a protocol be provided for patient monitoring on the inpatient and Ambulatory surgery units to include the continued use of the NPS and the POSS.

\section{Recommendation 13:}

If a patient experiences a POSS of 3 then it is recommended to notify an anesthesiologist, the pain management team or any provider available. If Over-sedation or a POSS of 4 occurs, it is recommended to call anesthesia, protect the airway and provide Narcan slowly as per hospital protocol.

\section{Clinical Specialty: Surgery}

Intended Users: Advanced practice nurses, Physicians Assistants, Hospitalists, Surgeons, Anesthesiologists, PACU RN's, Surgical NP's and Surgical PA's and CRNA's.

Outcomes: Decreased Numeric pain scores, shorter interval between reports of pain and treatment of pain, proper titration of opiates to decrease pain and risk for adverse effects, Assessment of sedation (Which precedes respiratory adverse reactions), decreased morbidity and mortality, decreased risk of suffering from chronic pain, decreased surgical 
infections, improved patient satisfaction and improved outcomes, decreased need to escalate issues to experts. 